\title{
Candida parapsilosis Protects Premature Intestinal Epithelial Cells from Invasion and Damage by Candida albicans
}

\author{
Sara Gonia', Linda Archambault' ${ }^{2}$ Margaret Shevik', Marie Altendahl', Emily Fellows', \\ Joseph M. Bliss ${ }^{3}$, Robert T. Wheeler ${ }^{2,4}$ and Cheryl A. Gale ${ }^{1,5 *}$ \\ ${ }^{1}$ Department of Pediatrics, University of Minnesota, Minneapolis, MN, USA, ${ }^{2}$ Department of Molecular and Biomedical \\ Sciences, University of Maine, Orono, ME, USA, ${ }^{3}$ Department of Pediatrics, Women \& Infants Hospital of Rhode Island, \\ Warren Alpert Medical School of Brown University, Providence, RI, USA, ${ }^{4}$ Graduate School of Biomedical Sciences and \\ Engineering, University of Maine, Orono, ME, USA, ${ }^{5}$ Department of Genetics, Cell Biology and Development, University of \\ Minnesota, Minneapolis, MN, USA
}

OPEN ACCESS

Edited by:

Lewis Phillip Rubin, Texas Tech University Health

Sciences Center, USA

Reviewed by: Ulrich Herbert Thome, Leipzig University, Germany

Rick Auten,

ConeHealth, USA

*Correspondence:

Cheryl A. Gale

galex012@umn.edu

Specialty section: This article was submitted to Neonatology,

a section of the journal

Frontiers in Pediatrics

Received: 02 January 2017 Accepted: 03 March 2017

Published: 22 March 2017

Citation:

Gonia S, Archambault L, Shevik M, Altendahl M, Fellows E, Bliss JM, Wheeler RT and Gale CA (2017) Candida parapsilosis Protects Premature Intestinal Epithelial Cells from Invasion and Damage by Candida albicans.

Front. Pediatr. 5:54. doi: 10.3389/fped.2017.00054
Candida is a leading cause of late-onset sepsis in premature infants and is thought to invade the host via immature or damaged epithelial barriers. We previously showed that the hyphal form of Candida albicans invades and causes damage to premature intestinal epithelial cells (plECs), whereas the non-hyphal Candida parapsilosis, also a fungal pathogen of neonates, has less invasion and damage abilities. In this study, we investigated the potential for $C$. parapsilosis to modulate pathogenic interactions of C. albicans with the premature intestine. While a mixed infection with two fungal pathogens may be expected to result in additive or synergistic damage to plECs, we instead found that $C$. parapsilosis was able to protect plECs from invasion and damage by C. albicans. C. albicans-induced plEC damage was reduced to a similar extent by multiple different $C$. parapsilosis strains, but strains differed in their ability to inhibit $C$. albicans invasion of plECs, with the inhibitory activity correlating with their adhesiveness for C. albicans and epithelial cells. C. parapsilosis cell-free culture fractions were also able to significantly reduce $C$. albicans adhesion and damage to plECs. Furthermore, coadministration of $C$. parapsilosis cell-free fractions with $C$. albicans was associated with decreased infection and mortality in zebrafish. These results indicate that $C$. parapsilosis is able to reduce invasion, damage, and virulence functions of $C$. albicans. Additionally, the results with cellular and cell-free fractions of yeast cultures suggest that inhibition of pathogenic interactions between $C$. albicans and host cells by $C$. parapsilosis occurs via secreted molecules as well as by physical contact with the $C$. parapsilosis cell surface. We propose that non-invasive commensals can be used to inhibit virulence features of pathogens and deserve further study as a non-pharmacological strategy to protect the fragile epithelial barriers of premature infants.

Keywords: Candida albicans, Candida parapsilosis, fungal pathogenesis, premature infant, intestinal epithelium, zebrafish model system 


\section{INTRODUCTION}

Candida albicans and Candida parapsilosis are the leading causes of invasive fungal disease in premature infants (1), with the intestinal tract being an important site for Candida invasion (2-4). For example, life-threatening gastrointestinal tract diseases that occur in premature infants such as necrotizing enterocolitis and spontaneous intestinal perforation are highly associated with concurrent diagnoses of invasive candidiasis. C. albicans and C. parapsilosis, along with other fungi, are prevalent commensals of the intestinal tract of infants (5-7), with high amounts of Candida colonization within the intestine being correlated with an increased risk for invasive disease (8). Administration of prophylactic doses of fluconazole to infants has been shown to decrease Candida colonization of the intestinal tract as well as the incidence of invasive candidiasis in premature infants (9). Concern remains, however, regarding off-target effects of antimicrobial agents, in particular, the impact on the developing intestinal microbiome and longer-term health (10).

Candida albicans is capable of forming three primary morphologies: ovoid yeast cells, chains of elongated yeast cells known as pseudohyphae, and extremely elongated filamentous cells known as true hyphae. Most Candida species, including C. parapsilosis, exist as yeast or pseudohyphae; only C. albicans, Candida tropicalis, and C. dubliniensis have been observed to form true hyphae (11). The ability to undergo hyphal morphogenesis is associated with the ability of $C$. albicans to invade and damage various human epithelial and endothelial tissues $(12,13)$. In particular, our laboratory has shown that $C$. albicans hyphae, but not yeast forms, cause significant invasion and damage of premature intestinal epithelial cells (pIECs) $(14,15)$. Other Candida species that do not form hyphae, including C. parapsilosis, are essentially inert with respect to this epithelial cell line (14).

Mixed infections that include more than one pathogen often have additive or synergistic effects on pathogenesis or virulence features as compared to infection with a single microbe. With respect to $C$. albicans infections, coinfection of vaginal epithelial cells with C. albicans and C. glabrata has been observed to result in increased epithelial cell injury as compared to infection with either single species (16). In addition, intra-abdominal infection of C. albicans along with Staphylococcus aureus results in $100 \%$ mortality in mice, whereas the mono-microbial infections are avirulent (17). Some microbes, on the other hand, have been observed to have activities that confer protection from pathogenic features of $C$. albicans infections. For example, Pseudomonas aeruginosa produces phenazines that inhibit the formation of C. albicans biofilms in vitro (18), and probiotic bacteria of the Bifidobacterium and Lactobacillus genera appear to reduce Candida colonization in extremely premature infants, although their efficacy in reducing fungal sepsis has not yet been conclusively shown (19). Collectively, these observations support the idea that the particular behavior of a microbe toward commensalism versus pathogenesis can be influenced by the relative activity of neighboring microbes.

In this study, we tested the hypothesis that C. parapsilosis, the second leading cause of fungal sepsis in premature infants, modulates C. albicans-induced damage and invasion of pIECs in vitro, as well as in vivo using a zebrafish model of candidiasis. Together, our results add mechanistic insight into pathogenic interactions between $C$. albicans and the premature intestine and how these interactions might be prevented.

\section{MATERIALS AND METHODS}

\section{Fungal Growth Conditions and Preparation of Cell and Cell-Free Fractions for Assays}

Yeast strains (Table 1) were propagated and maintained as described previously (20). Strains were recovered from $15 \%$ glycerol stocks stored at $-80^{\circ} \mathrm{C}$ by plating onto Yeast Peptone Dextrose agar and incubating at $30^{\circ} \mathrm{C}$ overnight. Individual colonies were then suspended and grown in synthetic dextrose complete medium containing $2 \%$ glucose at $30^{\circ} \mathrm{C}$ overnight prior to assays being performed. Cell concentrations were determined microscopically using a hemacytometer. To obtain cell-free culture fractions, yeast cells were grown as described above, sub-cultured into $\mathrm{H} 4$ tissue culture medium at a concentration of $2 \times 10^{6}$ cells $/ \mathrm{mL}$ and grown at $30^{\circ} \mathrm{C}$ for $12 \mathrm{~h}$. Yeast cells were pelleted by centrifugation at 13,000 rpm for $3 \mathrm{~min}$. The supernatants were removed carefully using a pipet, so as not to disturb the cell pellet. Supernatants were visualized microscopically using $60 \times$ magnification in multiple random fields to ensure that no yeast cells were present.

\section{pIEC Culture and Maintenance}

Primary premature human enterocytes (cell line H4) were cultivated and maintained using $\mathrm{H} 4$ growth medium and conditions as previously described (23).

\section{Epithelial Cell Damage (Cytotoxicity) Assay}

Premature intestinal epithelial cell damage was assessed as previously described $(14,24)$. Briefly, pIECs were cultured at a concentration of $2 \times 10^{4}$ cells/well and grown to approximately $80 \%$ confluence in a 96 -well flat-bottomed tissue culture plate (BD Biosciences, San Jose, CA, USA). The pIECs were incubated with yeast $\left(1 \times 10^{6} \mathrm{C}\right.$. albicans and/or $2 \times 10^{6} \mathrm{C}$. parapsilosis, both in $\sim 10 \mu \mathrm{L}$ volume $)$, C. albicans $\left(1 \times 10^{6}\right.$ cells $)$ with addition of C. parapsilosis cell-free culture fraction from $2 \times 10^{6}$ cells, or C. parapsilosis cell-free culture fraction alone. After $8 \mathrm{~h}$ of incubation, the amount of lactate dehydrogenase released from injured epithelial cells was measured using the Cyto-Tox-96 ${ }^{\circledR}$ assay

\begin{tabular}{llc}
\hline \multicolumn{2}{l}{ TABLE 1 } & \\
\hline Strain & Strains used in this study. & Source \\
\hline Ca SC5314 & Candida albicans laboratory strain & $(21)$ \\
Cp 4175 & Candida parapsilosis clinical isolate, neonate, cardiac & $(14)$ \\
& mass & \\
Ca A022b & C. albicans clinical isolate, neonate, pleural fluid & $(14)$ \\
Cp LOW & C. parapsilosis JMB72 & $(22)$ \\
Cp HIGH & C. parapsilosis JMB77 & $(22)$ \\
Ca GFP & C. albicans SC5314 ENO1:GFP-NAT1/ENO1 & J. Berman \\
& & $(U$ of MN)
\end{tabular}


(Promega, Madison, WI, USA) per the manufacturer's instructions. The amount of cell damage is represented as a percent of the control group for each comparison and the reported results are the averages of three independent experiments, each performed in triplicate. Statistical analyses were performed as follows. For two group comparisons, Student's $t$-test (pairing the data by day) was used. For three or more group comparisons, a blocked ANOVA was employed to account for day-to-day variation in pIEC passage number followed by post hoc separation of means using Tukey's HSD.

\section{Invasion Assay}

Candida albicans penetration of pIECs was determined as described previously $(14,15,24)$. In general, the yeast morphologic form is non-invasive whereas the hyphal form is capable of penetration with respect to H4 pIECs $(14,15)$. Hyphal cells were determined to be invading or not invading pIECs after $3 \mathrm{~h}$ of infection with C. albicans, in the presence or absence of C. parapsilosis or C. parapsilosis supernatant, using an immunocytochemical method. Briefly, non-invading fungal cells are labeled fluorescently via the Alexa 568 fluorophore, whereas invading fungal cells are inaccessible to the primary antibody and are not labeled. Approximately 30 fungal cells were analyzed for each experimental condition and day. Statistical analysis was performed using Student's $t$-test, pairing the data by day to account for pIEC passage number.

\section{Adhesion Assay}

Fungal cell adhesion to pIECs was determined as described previously (25). Briefly, pIECs were inoculated into 96-well tissue culture microplates at a density of $2 \times 10^{4}$ cells/well in a final volume of $100 \mu \mathrm{L}$ and incubated overnight. The medium was aspirated, and pIECs were infected with $100 \mu \mathrm{L}$ of $1 \times 10^{6} \mathrm{C}$. albicans cells suspended in either $\mathrm{H} 4$ media or C. parapsilosis cell-free fractions and incubated for $3 \mathrm{~h}$ at $37^{\circ} \mathrm{C}$ with $5 \% \mathrm{CO}_{2}$. Following incubation, the wells were washed with phosphate-buffered saline (PBS), the cells were fixed with $4 \% p$-formaldehyde, and the yeast cells were stained with $0.5 \%$ crystal violet. The optical densities at $600 \mathrm{~nm}$ were determined using a spectrophotometer, and the results were expressed as the percent $C$. albicans adhesion inhibition as compared to $100 \%$ adhesion of C. albicans alone to pIECs. Results are reported as the average of three independent experiments, each with six replicates. Statistical analysis was performed using Student's $t$-test, pairing the data by day to account for pIEC passage number.

\section{Zebrafish Growth, Maintenance, and Infection}

All animal studies were carried out in accordance with the recommendations in the Guide for the Care and Use of Laboratory Animals of the National Institutes of Health. All animals were treated in a humane manner according to guidelines of the University of Maine IACUC as detailed in protocol number A2015-11-03. The UMaine IACUC/Ethics Committee approved this protocol. Animals were euthanized by tricaine overdose.
Infected animals were monitored twice daily for signs of infection and morbid animals were euthanized. Wild-type AB zebrafish were maintained as described previously (26). Zebrafish larvae were grown in $\mathrm{E} 3$ medium $(5.0 \mathrm{mM} \mathrm{NaCl}, 0.17 \mathrm{mM} \mathrm{KCl}, 0.33$ $\mathrm{mMCaCl}, 0.33 \mathrm{mM}$ MgSO 4, 2 mM HEPES, pH 7) plus $0.3 \mu \mathrm{g} / \mathrm{mL}$ methylene blue for the first $6 \mathrm{~h}$ post-fertilization, then switched to E3 supplemented with $10 \mu \mathrm{g} / \mathrm{mL}$ 1-phenyl-2-thiourea to suppress pigmentation.

The C. albicans CAF2.1-dTom-NATr strain was used for all experiments in zebrafish (26-28) and was grown and prepared for infections as described previously (26). Overnight cultures were washed three times in calcium- and magnesium-free PBS and yeast cell concentrations were determined microscopically using a hemocytometer. Cell suspensions were adjusted to a concentration of $5 \times 10^{7}$ cells $/ \mathrm{mL}$ in $5 \%$ polyvinylpirrolidone dissolved in $\mathrm{H} 4$ media alone or fungal culture supernatants (described above in Section "Fungal Growth Conditions and Preparation of Cell and Cell-Free Fractions for Assays").

Zebrafish larvae were infected with yeast by injection into the swimbladder at 4 days post-fertilization (dpf) as previously described (29). Infected fish were individually screened at $2 \mathrm{~h}$ post-infection (hpi) on a Zeiss Axiovision Vivatome microscope. Mock-infected and infected fish were divided randomly into two cohorts. One cohort of 10 fish was held for 4 days, with counting and removal of deceased fish each day. The second cohort of fish was rescreened at 24 hpi and infection parameters (swimbladder deflation and epithelial breaching) were recorded. Statistical analysis was performed on GraphPad Prism version 7.00 for Mac (GraphPad Software, La Jolla, CA, USA, www.graphpad.com). Survival curves were analyzed using a log-rank (Mantel-Cox) test. Fisher's exact test, with Bonferroni correction for multiple tests, was used to detect differences in infection parameters among groups.

\section{RESULTS}

\section{pIEC Damage and Invasion by C. albicans Is Reduced by C. parapsilosis}

To determine the extent to which C. parapsilosis is able to modify host cell injury induced by $C$. albicans infection, pIEC damage was measured in the presence and absence of C. parapsilosis. Incubation of a mix of $C$. albicans and C. parapsilosis cultures (1:2 ratio) with pIECs caused significantly less damage as compared to infection with C. albicans alone (Figure 1A). By itself, C. parapsilosis strain 4175 does not damage pIECs (Figure 1A), consistent with the results of our previous study (14). C. parapsilosis had the same effect of decreasing pIEC damage caused by two different C. albicans strains (Figure 1A), a common laboratory strain (SC5314) and a clinical isolate (A022b), both previously shown to significantly damage pIECs (14).

During incubation of pIECs with C. albicans, yeast cells form elongated hyphae that start to penetrate the host cells after $\sim 3 \mathrm{~h}$; C. parapsilosis cells, which do not form hyphae, exhibit minimal invasion of pIECs (14). In a mixed infection of pIECs using C. parapsilosis and C. albicans, we found a reduction in the number of $C$. albicans hyphae able to penetrate pIECs as compared 
to infection with C. albicans alone (Figure 1B). The efficiency of C. albicans hyphal morphogenesis in the presence or absence of C. parapsilosis did not differ, thus the reduction in invasion was not due to differences in hyphal formation by C. albicans (data not shown). We also observed that C. parapsilosis cells tended to localize along the surface of $C$. albicans hyphae, raising the possibility that physical interactions between the fungal species were inhibiting $C$. albicans from interacting with and injuring host cells.

\section{C. parapsilosis Cell-Free Culture Fraction Reduces C. albicans-Induced pIEC Damage}

To determine if the C. parapsilosis activity that reduces pIEC damage and/or invasion by $C$. albicans is contained in the cellfree culture fraction, C. parapsilosis culture supernatants were used to resuspend C. albicans cells prior to infection of pIECs. Supernatants were harvested from $C$. parapsilosis cultures grown to the same density $\left(2 \times 10^{6}\right.$ cells $\left./ \mathrm{mL}\right)$ as those used in the invasion and damage assays (Figure 1) to ensure consistent

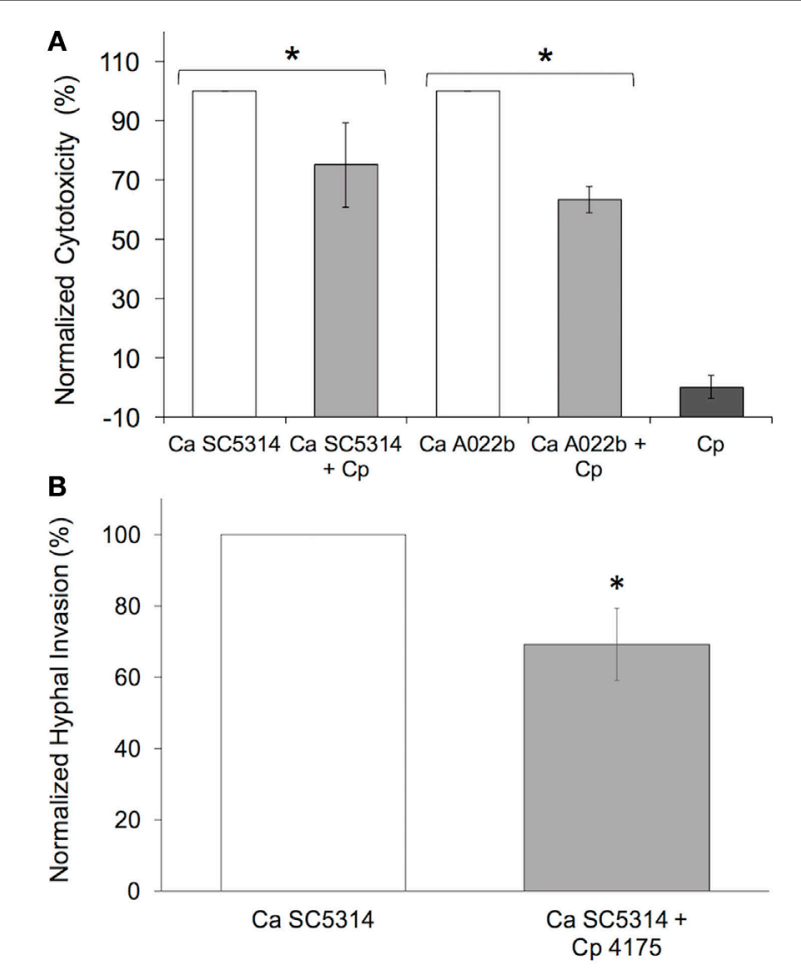

FIGURE 1 | Candida parapsilosis reduces premature intestinal epithelial cell damage (A) and invasion (B) by $C$. albicans strains. (A) Cell damage (lactate dehydrogenase amount) is plotted as a percentage of that caused by Candida albicans (Ca) strains (SC5314 and A022b) alone ("normalized cytotoxicity"). Cp, C. parapsilosis strain 4175. (B) C. albicans

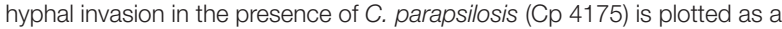
percentage of that observed for C. albicans (Ca SC5314) alone. Data shown are the average of three individual experiments. For both panels $(\mathbf{A}, \mathbf{B})$, an asterisk $\left(^{*}\right)$ indicates a statistically significant difference with $p \leq 0.05$. Error bars represent SEM. quantities of supernatant components between experiments. For pIEC invasion by $C$. albicans hyphae, addition of C. parapsilosis supernatants had no apparent effect, either in reducing or enhancing invasion (data not shown). For pIEC damage, C. albicans cells resuspended in C. parapsilosis supernatants had significantly reduced ability to damage pIECs, although not as much as when $C$. parapsilosis cells were also present ( $\sim 20 \%$ reduction for supernatants alone; $\sim 50 \%$ reduction for supernatants along with cells) (Figure 2). Control C. albicans cells that were resuspended in conditioned medium (incubated overnight at $37^{\circ} \mathrm{C}$ ) that was not exposed to C. parapsilosis were able to damage pIECs to the same extent as observed previously for unconditioned media (data not shown). These results suggest that $C$. parapsilosis cells secrete a factor that interferes with the ability of $C$. albicans to damage pIECs and that $C$. parapsilosis cells themselves also contribute to reducing damage to pIECs. Of note, C. parapsilosis supernatants had no effect on C. albicans growth or ability to form hyphae (data not shown). Thus, its function in reducing $C$. albicans damage of pIECs appears to occur independently of these two virulence factors.

\section{C. parapsilosis Reduces the Ability of C. albicans to Adhere to pIECs}

Fungal adhesion to host cells is thought to be an early step in the pathogenesis of $C$. albicans infections $(30,31)$. To determine how the C. parapsilosis cell-free fraction affects C. albicans adhesion to pIECs, we again used supernatants harvested from C. parapsilosis cultures as described above. Addition of $C$. parapsilosis supernatants caused an $\sim 15 \%$ reduction in the number of $C$. albicans cells able to adhere to pIECs $(p<0.05)$. Thus, the decreased pIEC damage from addition of $C$. parapsilosis supernatants correlates with reduced $C$. albicans adhesion to pIECs.

Most adhesion assays, including the one employed by us as described above, utilize a cell staining method to quantify yeast

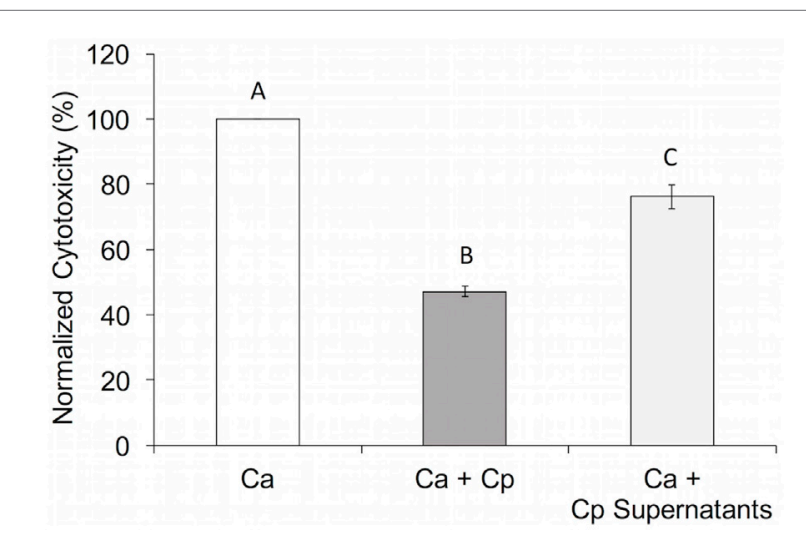

FIGURE 2 | Candida parapsilosis cell-free culture fraction reduces Candida albicans-induced premature intestinal epithelial cell damage. Cell damage (lactate dehydrogenase amount) is plotted as a percentage of that caused by C. albicans (Ca SC5314) alone ("normalized cytotoxicity"). Data shown are the average of three individual experiments. Letters are used to indicate statistical significance, where letters differ, $p<0.05$. Error bars represent SEM. 
cells spectrophotometrically. These methods cannot distinguish cells of different yeast species from each other, thus we are not able to determine the effect of $C$. parapsilosis cells on the adhesion of C. albicans to pIECs. As an alternative, we explored the extent to which adhesiveness of $C$. parapsilosis cells contributes to reducing pIEC damage caused by $C$. albicans by comparing two C. parapsilosis strains that differ with respect to adhesion to human FaDu epithelial cells (22). By themselves, both of these C. parapsilosis strains caused a similar, low amount of damage to pIECs that did not differ from that observed with C. parapsilosis strain 4175 (Figure 3A). Both strains were able to reduce C. albicans-induced pIEC damage (similar to strain 4175) and did not differ with respect to their specific activities. Thus, adhesiveness to $\mathrm{FaDu}$ cells does not correlate with differences in the ability of $C$. parapsilosis to inhibit pIEC damage by $C$. albicans. By contrast, pIEC invasion by $C$. albicans hyphae was reduced by coincubation with C. parapsilosis strain 4175 (Figure 1B) and the FaDu high-adhesion strain (Figure 3B),

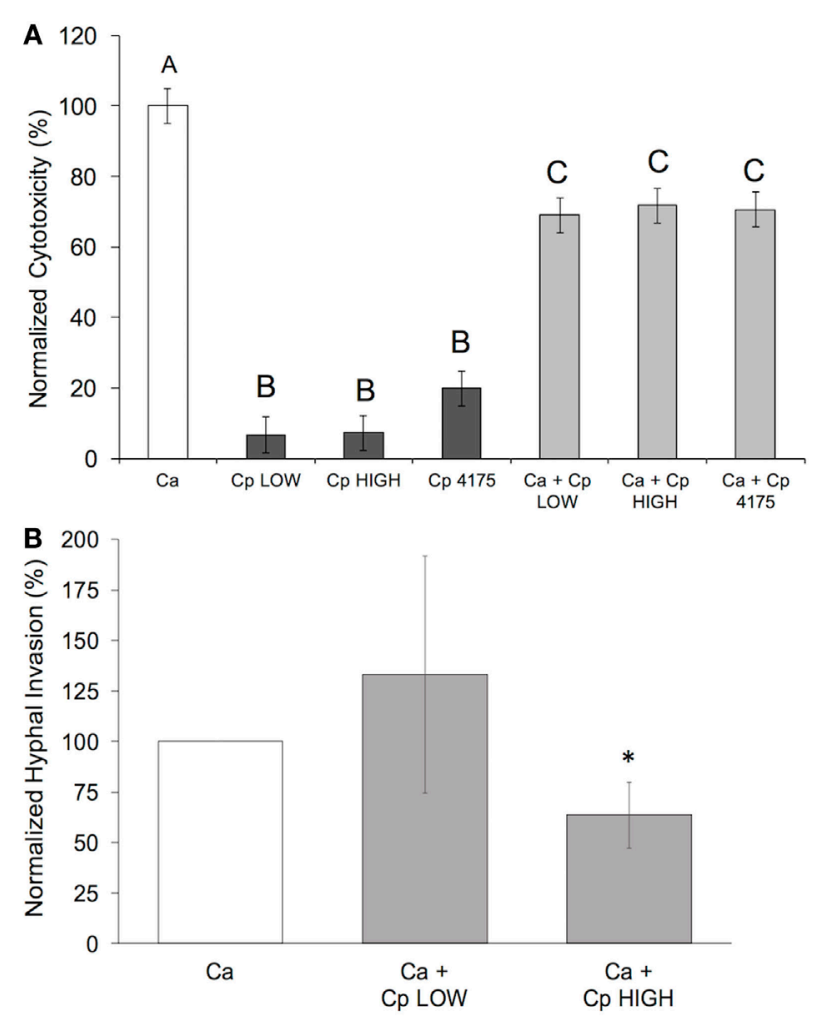

FIGURE 3 | Adhesiveness of Candida parapsilosis strains correlates with ability to reduce Candida albicans invasion, but not damage, of pIECs. (A) Cell damage (lactate dehydrogenase amount) is plotted as a percentage of that caused by C. albicans (Ca SC5314) alone ("normalized cytotoxicity"). Data shown are the average of three individual experiments. Letters are used to indicate statistical significance, where letters differ, $p<0.05$. Error bars represent SEM. (B) C. albicans hyphal invasion in the presence of $C$. parapsilosis $(\mathrm{Cp})$ strains is plotted as a percentage of that observed for C. albicans (Ca SC5314) alone. Data shown are the average of

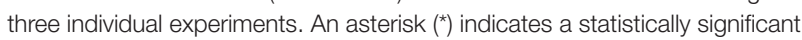
difference with $p \leq 0.05$. Error bars represent SEM. but not with the low-adhesion strain (Figure 3B). Furthermore, both strain 4175 and the $\mathrm{FaDu}$ high-adhesion strain were observed to localize along C. albicans hyphae whereas the $\mathrm{FaDu}$ low-adhesion strain was not (Figure 4). Thus, adhesiveness of C. parapsilosis strains correlates with their abilities to interact with $C$. albicans hyphae and to reduce $C$. albicans invasion of pIECs.

\section{C. parapsilosis Supernatants Protect Zebrafish from Infection by C. albicans}

The zebrafish swimbladder is a mucosal organ that is used for buoyancy and is both physiologically and ontologically most closely related to the mammalian lung (Figure 5A) (32-34). To test the protective ability of $C$. parapsilosis in vivo, zebrafish were infected in the swimbladder with $C$. albicans yeast suspended in C. parapsilosis supernatant, C. albicans supernatant, or unconditioned medium. This infection model recapitulates a number of aspects of in vitro C. albicans-epithelial interactions, permits mucosal infection in the context of a vertebrate immune system and allows for high inoculum doses that cause infection without immunosuppression $(28,29)$. Therefore, this optically transparent disease model is more complex than in vitro challenge of epithelial

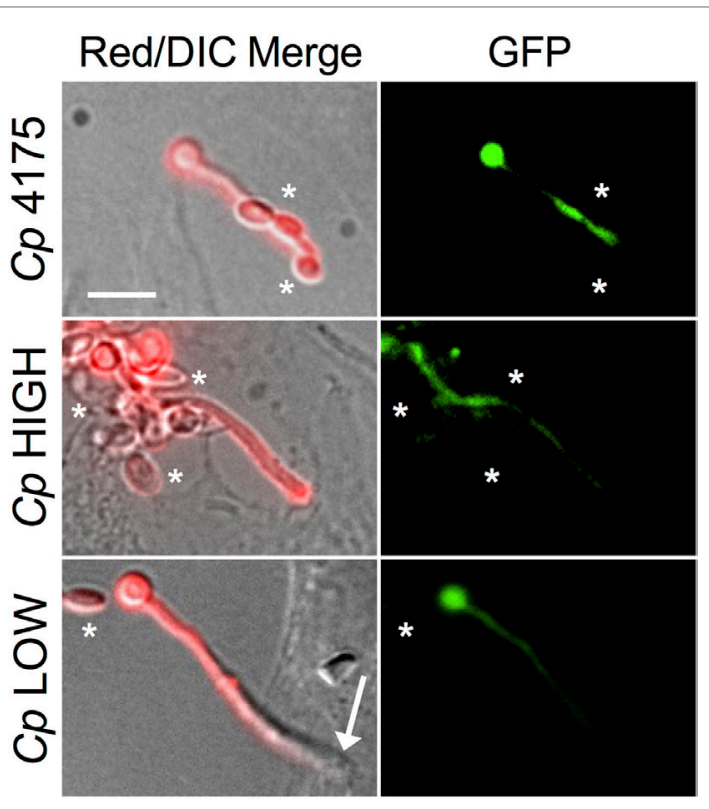

FIGURE 4 | Candida albicans and Candida parapsilosis interactions on premature intestinal epithelial cells (pIECs). Representative photomicrographs of $C$. albicans (Ca GFP) coincubated with C. parapsilosis $(C p)$ strains on plECs. A C. albicans strain expressing GFP was used to differentiate between C. albicans (red and green fluorescence) and C. parapsilosis (red fluorescence, no green fluorescence, see asterisks in GFP panels) yeast cells. Alexa 568 conjugated to phalloidin was used to stain fungal cells with red fluoroscence, as previously described (14). A penetrating C. albicans hypha lacks red fluorescent signal (Cp LOW, Red/DIC Merge panel, arrow). Non-penetrating Candida cells exhibit red fluorescent signal. Cp 4175 and $\mathrm{Cp} \mathrm{HIGH}$ cells make contacts with C. albicans hyphae (top and center merge panels, asterisks), while Cp LOW cells do not (bottom merge panel, asterisk). Scale bar, $10 \mu \mathrm{m}$. 
A
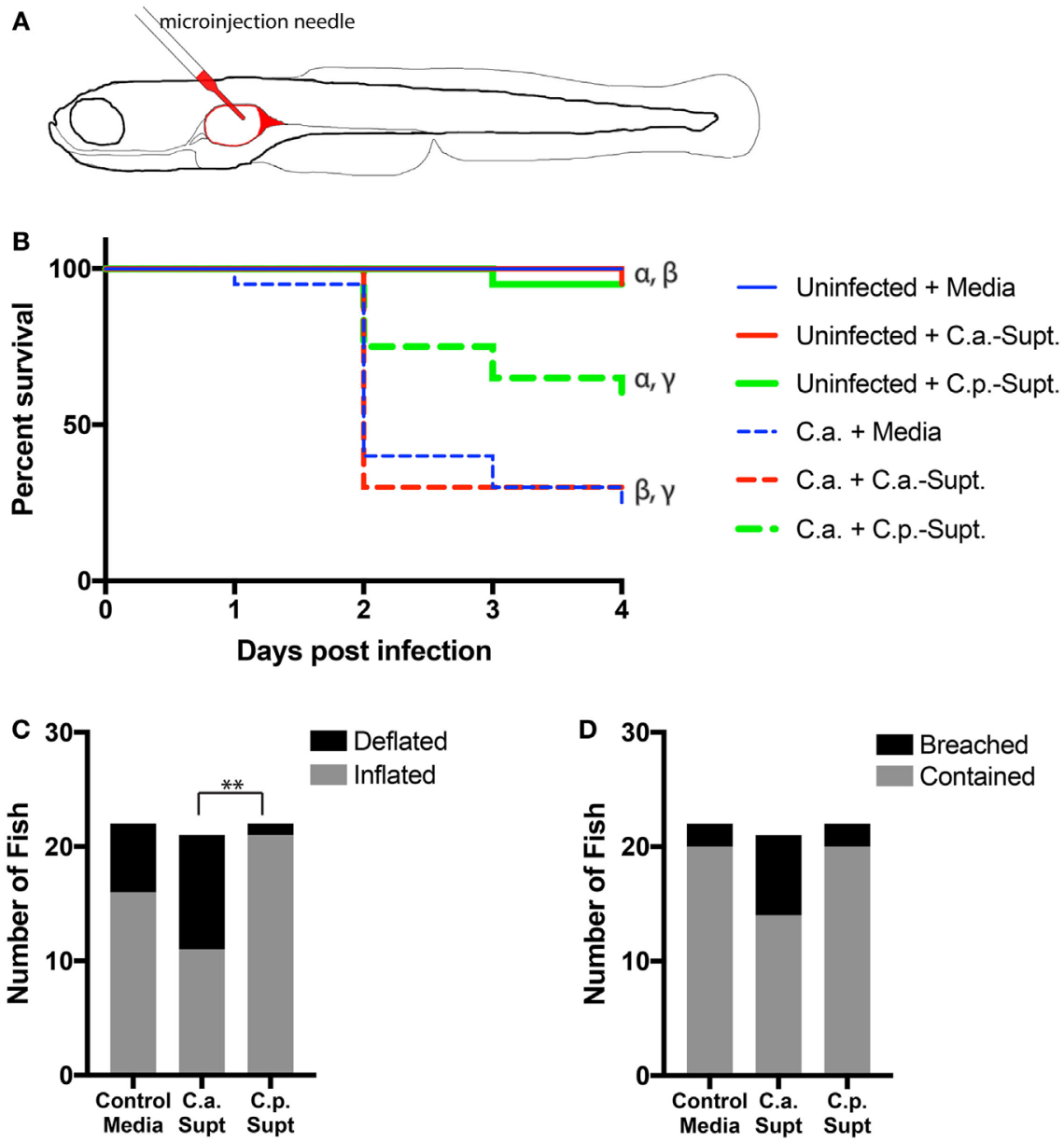

FIGURE 5 | Candida parapsilosis cell-free culture fraction protects zebrafish from infection by Candida albicans. (A) Schematic of infection model. The larval zebrafish swimbladder offers a transparent vertebrate mucosal infection model that is amenable to non-invasive imaging of both the host and the pathogen. (B-D) Zebrafish at 4 days post-fertilization with inflated swimbladders were infected in their swimbladders by glass needle injection with $C$. albicans yeast cells (C.a.) suspended in control (H4) media or in supernatants from C. parapsilosis (C.p. Supt) or C. albicans (C.a. Supt.) cultures. (B) Relative survival of fish infected with C. albicans with or without Candida supernatants. All C. albicans-infected fish cohorts are significantly different from their respective controls. C. parapsilosis supernatants significantly reduce the mortality of a $C$. albicans infection (denoted by $\gamma$ ). Matching Greek letters label individual comparisons: $\alpha, p<0.01 ; \beta$, $p<0.0001 ; \gamma, p<0.05$. Survival data are pooled from two independent experiments, $n=20$ per group. All pair-wise comparisons were made with the Mantel-Cox test. (C,D) Fish from the experiment in (B) were viewed by fluorescence microscopy at $24 \mathrm{~h}$ post-infection and scored for two indicators of infection, swimbladder deflation $\left[(\mathbf{C}){ }^{* *} p<0.01\right]$ and breaching of epithelial barrier (D). Examples of these phenotypes are shown in Figure S1 in Supplementary Material. Data were pooled from two independent experiments and analyzed by Fisher's exact test with Bonferroni correction (Control media, $n=22$, C.a. supernatant, $n=21$, C.p. supernatant, $n=22$ ).

cells but is not as complex as the mouse intestinal colonization model, which is reliant on antibiotic treatment for colonization and requires both physical disturbance and immunosuppression to yield lethal infection (35). Cell-free fractions of C. albicans and C. parapsilosis cultures, as well as medium alone, had no effect on zebrafish viability. When C. albicans was suspended in C. parapsilosis supernatant, mortality was significantly reduced (Figure 5B) as compared to C. albicans suspended in its own supernatant or fresh unconditioned medium. The effect of C. parapsilosis cells along with cell-free fractions on zebrafish viability could not be tested due to the number of yeast in the mixed inoculum being beyond the physical constraints of microinjection into the zebrafish swimbladder.
Deflation of the swimbladder and breaching of the swimbladder epithelium by hyphae are visual hallmarks of C. albicans infection in zebrafish (29). Nearly half of the fish infected with C. albicans suspended in C. albicans supernatant experienced swimbladder deflation at 24 hpi. By contrast, significantly fewer fish had deflated swimbladders when C. albicans was suspended in C. parapsilosis supernatants (Figure 5C). In addition, there was a trend toward more breaching of the swimbladder epithelium by $C$. albicans hyphae with addition of $C$. albicans supernatant than for C. parapsilosis supernatant or control media, although this difference did not reach statistical significance (Figure 5D). Together, these results indicate that $C$. parapsilosis supernatants protect zebrafish from the effects of $C$. albicans infection. 


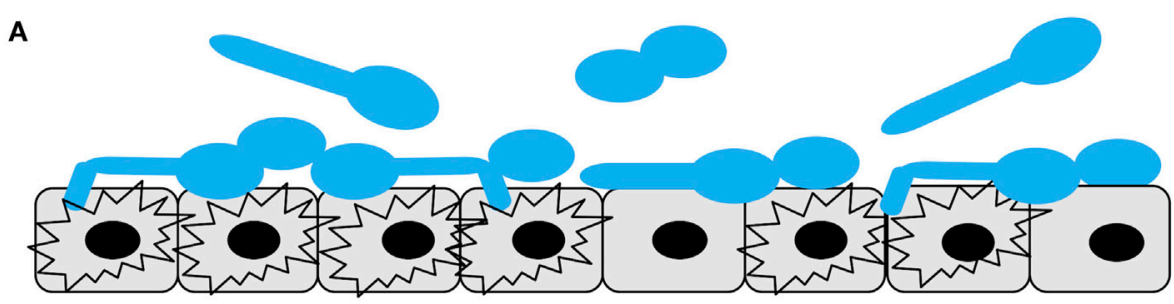

B

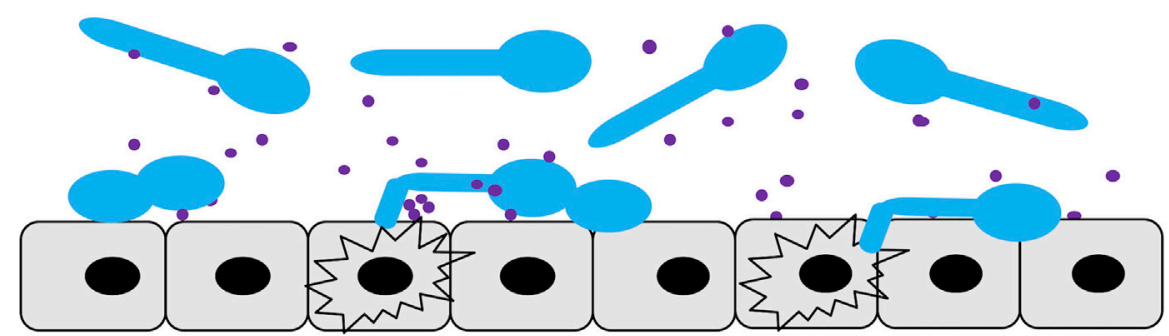

C

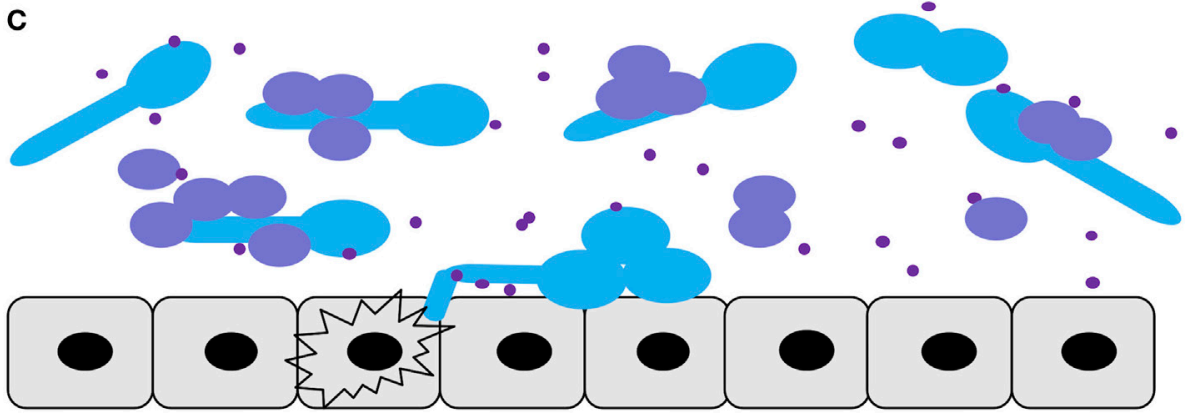

FIGURE 6 | Diagram summarizing the Candida-premature intestinal epithelial cell (pIEC) interactions described in this study. Infection models: (A) Candida albicans alone, (B) C. albicans along with Candida parapsilosis cell-free culture fraction, (C) mix of C. albicans and C. parapsilosis cultures. For all panels: C. albicans yeast and hyphal cells, light blue; C. parapsilosis cells, purple; C. parapsilosis cell-free culture factors, purple dots; plECs, gray; damaged plECs, black explosion outlines.

\section{DISCUSSION}

Three epithelial cell infection models were compared in this study (Figure 6): C. albicans alone (Figure 6A), C. albicans with the addition C. parapsilosis cell-free fractions (supernatants) (Figure 6B), and a mix of C. albicans and C. parapsilosis cultures (Figure 6C). Comparison of C. parapsilosis cellular versus cellfree culture fractions allowed us to study the relative contributions of each and gain mechanistic insight with respect to pathogenic interactions of C. albicans with pIECs. The amount of Candida cells that we used for infection of pIECs $\left(\sim 10^{5}\right.$ cells $\left./ \mathrm{mL}\right)$ is within the range of colonization levels that have been reported in a few studies of fecal fungal colonization of infants and children $\left(\sim 10^{1}-10^{8}\right.$ cells $\left./ \mathrm{mL}\right)$, with the higher levels being more typical for subjects with concurrent diarrhea $(5,36,37)$. Thus, we think that our infection models are within a physiologically relevant range. Although infection with two fungal pathogens may be expected to cause more host cell damage, we found that C. parapsilosis reduces pathogenic interactions of $C$. albicans with host cells. This is consistent with the idea that pathogens can behave as commensals if they coexist with other microbes that are capable of modulating their virulence functions. Conversely, it is also possible that pathogens could exhibit heightened virulence functions depending on the neighboring microflora. It is notable that the zebrafish swimbladder is not sterile, so the conservation of a protective effect in this mucosal model suggests that this work has relevance in vivo. As is true for all studies with infection models, the effects on virulence that we observe must be tested and corroborated in further animal and, eventually, human studies before being brought to the clinic.

Candida parapsilosis supernatants alone were able to reduce C. albicans adhesion to, and damage of, pIECS (Figure 6B), and occurred independent of effects on C. albicans growth or hyphal formation. It seems reasonable that $C$. albicans would need to establish and maintain close contact with pIECs in order to damage them via physical penetration and/or via targeted delivery of membrane-degrading enzymes such as secreted aspartyl proteinases (SAPs) or candidalysin (38-40). The result that C. parapsilosis supernatants reduce pIEC damage by C. albicans, then, may be related to their ability to reduce $C$. albicans contacts with host cells, potentially via competitive blockade of hydrophobic contacts or fungal adhesin-host receptor interactions (41). 
In addition, or alternatively, C. parapsilosis supernatants may contain a factor that inhibits $C$. albicans secreted enzymes (e.g., SAPs) that degrade pIEC membrane components or that alter the biology of the pIECs (e.g., downregulate a receptor) such that they are less susceptible to adhesion and damage. Addition of C. parapsilosis cells along with their supernatants had a greater protective effect against $C$. albicans-induced pIEC damage than with the addition of supernatants alone (Figure $6 \mathrm{C}$ ). The result that there is an additive effect of $C$. parapsilosis cell and cell-free fractions suggests that each of them is acting via different inhibition mechanisms.

It seems likely that fungal-host cell contact would also be important for C. albicans to successfully invade pIECs. In support of this mechanistic step, when C. parapsilosis cells plus supernatants were coincubated with C. albicans, only the C. parapsilosis strains that were seen to localize along $C$. albicans hyphae were able to reduce C. albicans invasion of pIECs. C. parapsilosis supernatants by themselves also reduce the ability of $C$. albicans cells to adhere to pIECs; however, they appeared to have no effect on C. albicans invasion of pIECs. Importantly, the invasion assay employed here only analyzes the invasion efficiency of those fungal cells that are already in contact with pIECs (in the same microscopic plane of view). No conclusion can be drawn, then, about the connection between adhesion and invasion using this particular assay. We think it likely that, in the presence of C. parapsilosis supernatant, more $C$. albicans cells never made contact with pIECs and were washed away during the invasion assay. Consistent with a protective effect of C. parapsilosis supernatants in inhibiting C. albicans invasion of epithelia, although not statistically significant, cell-free fractions tended to reduce the ability of $C$. albicans to penetrate the epithelial lining of zebrafish swimbladders (Figure 5D). Thus, our collective data suggest that $C$. albicans invasion and injury of pIECs can be prevented by both $C$. parapsilosis cell and cellfree fractions (Figures 6B,C), either partially or completely, by inhibiting C. albicans adhesion to the host cell.

Two mechanisms have been described for C. albicans invasion of adult intestinal epithelial cells: active, physical penetration by hyphae, and endocytosis induced by protein-protein interactions (12). For pIECs, our laboratory has observed that the primary mechanism of invasion is penetration by hyphae; only live, actively elongating hyphae are capable of penetrating pIECs (Sara Gonia and Cheryl A. Gale, unpublished data). The identity of the specific factors and processes that mediate hyphal invasion of pIECs remain unknown, but fungal adhesion, physical forces, and targeted secretion of host membrane-degrading enzymes (e.g., proteinases, lipases, candidalysin) from the hyphal tip are likely to be involved as these features have been shown to facilitate invasion of other human cell lines $(31,38)$.

Cell-free fractions from the yeast Saccharomyces boulardii also appear to have a protective effect with respect to pathogenic interactions of $C$. albicans with human intestinal epithelial cells. $S$. boulardii supernatants reduce adhesion of $C$. albicans to adult Caco- 2 cells and this is associated with decreased IL-8 cytokine release by enterocytes (25). Saccharomyces species are typically regarded as non-pathogenic for the majority of individuals, except in rare cases of severe immunosuppression (42). C. parapsilosis, on the other hand, is regarded as a pathogen, particularly in infants $(1,43)$. Thus, its role in protection against C. albicansinduced mortality in zebrafish, and injury and invasion of epithelia in vitro, seems somewhat counter-intuitive. Of note, although the same C. parapsilosis clone has been isolated from both the intestinal tract and blood of premature infants with fungal sepsis (4), C. parapsilosis consistently causes minimal to no damage of pIECs [Figures 1 and 5; (14)]. These observations suggest that C. parapsilosis gains entry to the host via alternative sites [e.g., intravascular catheters and endothelium (44)] and support the idea that pathogenesis mechanisms employed by Candida species are host site- and species-specific (12).

In summary, C. parapsilosis reduces C. albicans-induced injury and invasion of pIECs and these effects are at least partially due to the ability of both secreted and cellular C. parapsilosis factors to reduce $C$. albicans physical interactions with host cells. Further studies are needed to understand the molecules on the surface of $C$. parapsilosis as well as the secreted factors that inhibit C. albicans adhesion to, and injury of, pIECs. Potential cell-surface candidates include homologs of C. albicans Als proteins and other adhesins $(45,46)$. For secreted factors, identification of molecules in supernatant fractions that confer inhibitory activities will provide additional mechanistic insight. Ultimately, a more complete understanding of microbe-microbe and host-microbe interactions that are associated with protection from disease may lead to the development of therapeutic strategies that promote intestinal health in at-risk patient populations.

\section{AUTHOR CONTRIBUTIONS}

SG designed and performed experiments, analyzed data, and wrote the manuscript draft. LA designed and performed experiments, analyzed data, assisted in manuscript writing, and revised the manuscript. MS, MA, and EF performed experiments, analyzed data, and revised the manuscript. JB assisted in development of the project, data analysis, and writing and revision of the manuscript. RW assisted in development of the project, designing of experimental approach, data analysis, and manuscript writing, and revised the manuscript. CG conceived the project, assisted in experimental design, data analysis, and writing of the manuscript, and revised the manuscript.

\section{ACKNOWLEDGMENTS}

The authors thank Judith Berman for providing strain Ca GFP, Emma Filtz, Rebekah Jensen, Colleen Jodarski, Kayla Dharampaul, and Veronika Rybarova for technical assistance and Tim Heisel for helpful discussions and critical review of the manuscript. This work was supported by NIH awards R01AI057440 (CG), P20RR018728 and P20GM103537 (JB), and R15AI094406 (RW). $\mathrm{RW}$ is a Burroughs Wellcome Investigator.

\section{SUPPLEMENTARY MATERIAL}

The Supplementary Material for this article can be found online at http://journal.frontiersin.org/article/10.3389/fped. 2017.00054/full\#supplementary-material. 
VIDEO S1 | Animated Z-stack of Figure S1A in Supplementary Material. Z-stack of a representative fish with partial swimbladder deflation and epithelia invasion. In fish like this representative, the swimbladder retains its air bubble (black oval). Candida albicans hyphae (purple) fill the distended space behind the air bubble and grow along the epithelial lining of the swimbladder. Filaments can be seen penetrating the epithelial barrier and invading the tissue under and behind the swimbladder. The animation begins near the midline of the fish and moves outward to the skin. Interslice interval $=5 \mu \mathrm{m}$. Scale bar $=150 \mu \mathrm{m}$.

\section{REFERENCES}

1. Chow BD, Linden JR, Bliss JM. Candida parapsilosis and the neonate: epidemiology, virulence and host defense in a unique patient setting. Expert Rev Anti Infect Ther (2012) 10(8):935-46. doi:10.1586/eri.12.74

2. Coates EW, Karlowicz MG, Croitoru DP, Buescher ES. Distinctive distribution of pathogens associated with peritonitis in neonates with focal intestinal perforation compared with necrotizing enterocolitis. Pediatrics (2005) 116(2):e241-6. doi:10.1542/peds.2004-2537

3. Ragouilliaux CJ, Keeney SE, Hawkins HK, Rowen JL. Maternal factors in extremely low birth weight infants who develop spontaneous intestinal perforation. Pediatrics (2007) 120(6):e1458-64. doi:10.1542/peds.2006-2804

4. Saiman L, Ludington E, Pfaller M, Rangel-Frausto S, Wiblin RT, Dawson J, et al. Risk factors for candidemia in neonatal intensive care unit patients. The national epidemiology of mycosis survey study group. Pediatr Infect Dis J (2000) 19(4):319-24. doi:10.1097/00006454-200004000-00011

5. Heisel T, Podgorski H, Staley CM, Knights D, Sadowsky MJ, Gale CA. Complementary amplicon-based genomic approaches for the study of fungal communities in humans. PLoS One (2015) 10(2):e0116705. doi:10.1371/ journal.pone.0116705

6. Kaufman DA, Gurka MJ, Hazen KC, Boyle R, Robinson M, Grossman LB. Patterns of fungal colonization in preterm infants weighing less than 1000 grams at birth. Pediatr Infect Dis J (2006) 25(8):733-7. doi:10.1097/ 01.inf.0000226978.96218.e6

7. Strati F, Di Paola M, Stefanini I, Albanese D, Rizzetto L, Lionetti P, et al. Age and gender affect the composition of fungal population of the human gastrointestinal tract. Front Microbiol (2016) 7:1227. doi:10.3389/fmicb.2016.01227

8. Pappu-Katikaneni LD, Rao KP, Banister E. Gastrointestinal colonization with yeast species and Candida septicemia in very low birth weight infants. Mycoses (1990) 33(1):20-3.

9. Kaufman D, Boyle R, Hazen KC, Patrie JT, Robinson M, Donowitz LG. Fluconazole prophylaxis against fungal colonization and infection in preterm infants. N Engl J Med (2001) 345(23):1660-6. doi:10.1056/NEJMoa010494

10. Vangay P, Ward T, Gerber JS, Knights D. Antibiotics, pediatric dysbiosis, and disease. Cell Host Microbe (2015) 17(5):553-64. doi:10.1016/ j.chom.2015.04.006

11. Thompson DS, Carlisle PL, Kadosh D. Coevolution of morphology and virulence in Candida species. Eukaryot Cell (2011) 10(9):1173-82. doi:10.1128/ EC.05085-11

12. Dalle F, Wachtler B, L'Ollivier C, Holland G, Bannert N, Wilson D, et al. Cellular interactions of Candida albicans with human oral epithelial cells and enterocytes. Cell Microbiol (2010) 12(2):248-71. doi:10.1111/j.1462-5822.2009. 01394.x

13. Phan QT, Myers CL, Fu Y, Sheppard DC, Yeaman MR, Welch WH, et al. Als3 is a Candida albicans invasin that binds to cadherins and induces endocytosis by host cells. PLoS Biol (2007) 5(3):e64. doi:10.1371/journal.pbio.0050064

14. Falgier C, Kegley S, Podgorski H, Heisel T, Storey K, Bendel CM, et al. Candida species differ in their interactions with immature human gastrointestinal epithelial cells. Pediatr Res (2011) 69(5 Pt 1):384-9. doi:10.1203/ PDR.0b013e31821269d5

15. Gonia S, Larson B, Gale CA. PCR-mediated gene modification strategy for construction of fluorescent protein fusions in Candida parapsilosis. Yeast (2016) 33(2):63-9. doi:10.1002/yea.3141

16. Alves CT, Wei XQ, Silva S, Azeredo J, Henriques M, Williams DW. Candida albicans promotes invasion and colonisation of Candida glabrata in a reconstituted human vaginal epithelium. J Infect (2014) 69(4):396-407. doi:10.1016/ j.jinf.2014.06.002

17. Nash EE, Peters BM, Fidel PL, Noverr MC. Morphology-independent virulence of Candida species during polymicrobial intra-abdominal infections
VIDEO S2 | Animated Z-stack of Figure S1B in Supplementary Material. Z-stack of a representative fish with complete swimbladder deflation and extensive invasion of epithelium. In fish like this representative, the swimbladder becomes completely deflated during infection. Here, many Candida albicans hyphae have breached the swimbladder epithelium, have penetrated the tissues surrounding the swimbladder, and are even growing through the skin. The animation begins near the midline of the fish and moves outward to the skin. Interslice interval $=5 \mu \mathrm{m}$. Scale bar $=150 \mu \mathrm{m}$.

with Staphylococcus aureus. Infect Immun (2015) 84(1):90-8. doi:10.1128/ IAI.01059-15

18. Morales DK, Grahl N, Okegbe C, Dietrich LE, Jacobs NJ, Hogan DA. Control of Candida albicans metabolism and biofilm formation by Pseudomonas aeruginosa phenazines. MBio (2013) 4(1):e526-512. doi:10.1128/mBio.00526-12

19. Hu HJ, Zhang GQ, Zhang Q, Shakya S, Li ZY. Probiotics prevent Candida colonization and invasive fungal sepsis in preterm neonates: a systematic review and meta-analysis of randomized controlled trials. Pediatr Neonatol (2016). doi:10.1016/j.pedneo.2016.06.001

20. Sherman F. Getting started with yeast. In: Guthrie C, Fink G, Abelson J, Simon M, editors. Methods Enzymol; Guide to Yeast Genetics and Molecular Biology. (Vol. 194), San Diego, CA: Harcourt Brace Jovanovich (1991). p. 3-20.

21. Gillum AM, Tsay EY, Kirsch DR. Isolation of the Candida albicans gene for orotidine- $5^{\prime}$-phosphate decarboxylase by complemetation of S. cerevisiae ura 3 and E. coli pyrF mutations. Mol Gen Genet (1984) 198(1):179-82. doi:10.1007/ BF00328721

22. Bliss JM, Wong AY, Bhak G, Laforce-Nesbitt SS, Taylor S, Tan S, et al. Candida virulence properties and adverse clinical outcomes in neonatal candidiasis. J Pediatr (2012) 161(3):441-7e2. doi:10.1016/j.jpeds.2012.02.051

23. Sanderson IR, Ezzell RM, Kedinger M, Erlanger M, Xu ZX, Pringault E, et al. Human fetal enterocytes in vitro: modulation of the phenotype by extracellular matrix. Proc Natl Acad Sci U S A (1996) 93(15):7717-22. doi:10.1073/ pnas.93.15.7717

24. Brand A, Vacharaksa A, Bendel C, Norton J, Haynes P, Henry-Stanley M, et al. An internal polarity landmark is important for externally induced hyphal behaviors in Candida albicans. Eukaryot Cell (2008) 7(4):712-20. doi:10.1128/ EC.00453-07

25. Murzyn A, Krasowska A, Augustyniak D, Majkowska-Skrobek G, Lukaszewicz M, Dziadkowiec D. The effect of Saccharomyces boulardii on Candida albicans-infected human intestinal cell lines Caco-2 and intestin 407. FEMS Microbiol Lett (2010) 310(1):17-23. doi:10.1111/j.1574-6968.2010.02037.x

26. Brothers KM, Newman ZR, Wheeler RT. Live imaging of disseminated candidiasis in zebrafish reveals role of phagocyte oxidase in limiting filamentous growth. Eukaryot Cell (2011) 10(7):932-44. doi:10.1128/EC.05005-11

27. Brothers KM, Gratacap RL, Barker SE, Newman ZR, Norum A, Wheeler RT. NADPH oxidase-driven phagocyte recruitment controls Candida albicans filamentous growth and prevents mortality. PLoS Pathog (2013) 9(10):e1003634. doi:10.1371/journal.ppat.1003634

28. Gratacap RL, Rawls JF, Wheeler RT. Mucosal candidiasis elicits NF-kappaB activation, proinflammatory gene expression and localized neutrophilia in zebrafish. Dis Model Mech (2013) 6(5):1260-70. doi:10.1242/dmm.012039

29. Gratacap RL, Bergeron AC, Wheeler RT. Modeling mucosal candidiasis in larval zebrafish by swimbladder injection. J Vis Exp (2014) 93:e52182. doi:10.3791/52182

30. Mayer FL, Wilson D, Hube B. Candida albicans pathogenicity mechanisms. Virulence (2013) 4(2):119-28. doi:10.4161/viru.22913

31. Wachtler B, Wilson D, Haedicke K, Dalle F, Hube B. From attachment to damage: defined genes of Candida albicans mediate adhesion, invasion and damage during interaction with oral epithelial cells. PLoS One (2011) 6(2):e17046. doi:10.1371/journal.pone.0017046

32. Robertson GN, McGee CA, Dumbarton TC, Croll RP, Smith FM. Development of the swimbladder and its innervation in the zebrafish, Danio rerio. J Morphol (2007) 268(121):967-85. doi:10.1002/jmor.10558

33. Winata CL, Korzh S, Kondrychyn I, Zheng W, Korzh V, Gong Z. Development of zebrafish swimbladder: the requirement of Hedgehog signaling in specification and organization of the three tissue layers. Dev Biol (2009) 331(2):222-36. doi:10.1016/j.ydbio.2009.04.035

34. Zheng W, Wang Z, Collins JE, Andrews RM, Stemple D, Gong Z. Comparative transcriptome analyses indicate molecular homology of zebrafish swimbladder 
and mammalian lung. PLoS One (2011) 6(8):e24019. doi:10.1371/journal. pone.0024019

35. Koh AY. Murine models of Candida gastrointestinal colonization and dissemination. Eukaryot Cell (2013) 12(11):1416-22. doi:10.1128/EC.00196-13

36. Forbes D, Ee L, Camer-Pesci P, Ward PB. Faecal Candida and diarrhea. Arch Dis Child (2001) 84(4):328-31. doi:10.1136/adc.84.4.328

37. Heyworth B, Brown J. Jejunal microflora in malnourished Gambian children. Arch Dis Child (1975) 50(1):27-33. doi:10.1136/adc.50.1.27

38. Moyes DL, Wilson D, Richardson JP, Mogavero S, Tang SX, Wernecke J, et al. Candidalysin is a fungal peptide toxin critical for mucosal infection. Nature (2016) 532(7597):64-8. doi:10.1038/nature17625

39. Naglik J, Albrecht A, Bader O, Hube B. Candida albicans proteinases and host/pathogen interactions. Cell Microbiol (2004) 6(10):915-26. doi:10.1111/j.1462-5822.2004.00439.x

40. Zhu W, Filler SG. Interactions of Candida albicans with epithelial cells. Cell Microbiol (2010) 12(3):273-82. doi:10.1111/j.1462-5822.2009.01412.x

41. Hofs S, Mogavero S, Hube B. Interaction of Candida albicans with host cells: virulence factors, host defense, escape strategies, and the microbiota. J Microbiol (2016) 54(3):149-69. doi:10.1007/s12275-016-5514-0

42. Chitasombat MN, Kofteridis DP, Jiang Y, Tarrand J, Lewis RE, Kontoyiannis DP. Rare opportunistic (non-Candida, non-Cryptococcus) yeast bloodstream infections in patients with cancer. J Infect (2012) 64(1):68-75. doi:10.1016/ j.jinf.2011.11.002

43. Trofa D, Gacser A, Nosanchuk JD. Candida parapsilosis, an emerging fungal pathogen. Clin Microbiol Rev (2008) 21(4):606-25. doi:10.1128/ CMR.00013-08
44. Shintaku T, Glass KA, Hirakawa MP, Longley SJ, Bennett RJ, Bliss JM, et al. Human endothelial cells internalize Candida parapsilosis via N-WASPmediated endocytosis. Infect Immun (2013) 81(8):2777-87. doi:10.1128/ IAI.00535-13

45. Bertini A, Zoppo M, Lombardi L, Rizzato C, De Carolis E, Vella A, et al. Targeted gene disruption in Candida parapsilosis demonstrates a role for CPAR2_404800 in adhesion to a biotic surface and in a murine model of ascending urinary tract infection. Virulence (2016) 7(2):85-97. doi:10.1080/ 21505594.2015.1112491

46. Kozik A, Karkowska-Kuleta J, Zajac D, Bochenska O, Kedracka-Krok S, Jankowska U, et al. Fibronectin-, vitronectin- and laminin-binding proteins at the cell walls of Candida parapsilosis and Candida tropicalis pathogenic yeasts. BMC Microbiol (2015) 15:197. doi:10.1186/s12866-015-0531-4

Conflict of Interest Statement: The authors declare that the research was conducted in the absence of any commercial or financial relationships that could be construed as a potential conflict of interest.

Copyright $\odot 2017$ Gonia, Archambault, Shevik, Altendahl, Fellows, Bliss, Wheeler and Gale. This is an open-access article distributed under the terms of the Creative Commons Attribution License (CC BY). The use, distribution or reproduction in other forums is permitted, provided the original author(s) or licensor are credited and that the original publication in this journal is cited, in accordance with accepted academic practice. No use, distribution or reproduction is permitted which does not comply with these terms. 